\title{
Paroxetine reduces crying in young women watching emotional movies
}

\author{
Frederik M. van der Veen • Joyce Jorritsma • \\ Carola Krijger • Ad J. Vingerhoets
}

Received: 8 March 2011 / Accepted: 31 August 2011 /Published online: 16 September 2011

(C) The Author(s) 2011. This article is published with open access at Springerlink.com

\begin{abstract}
Rationale Crying is a unique human emotional reaction that has not received much attention from researchers. Little is known about its underlying neurobiological mechanisms, although there is some indirect evidence suggesting the involvement of central serotonin.

Objectives We examined the acute effects of the administration of $20 \mathrm{mg}$ paroxetine on the crying of young, healthy females in response to emotional movies.

Methods We applied a double-blind, crossover randomised design with 25 healthy young females as study participants. On separate days, they received either paroxetine or placebo and were exposed to one of two emotional movies: 'Once Were Warriors' and 'Brian's Song'. Crying was assessed by self-report. In addition, the reactions to emotional International Affective Picture System (IAPS) pictures and mood were measured.

Results Paroxetine had a significant inhibitory effect on crying. During both films, the paroxetine group cried significantly less than the placebo group. In contrast, no
\end{abstract}

F. M. van der Veen $(\bowtie)$

Department of Psychiatry, Erasmus Medical Center,

PO Box 2040, 3000 CA Rotterdam, The Netherlands

e-mail: f.vanderveen@erasmusmc.nl

F. M. Veen

e-mail: veen@fsw.eur.nl

J. Jorritsma $\cdot$ C. Krijger · A. J. Vingerhoets

Clinical Psychology Section, Tilburg University,

Tilburg, The Netherlands

Present Address:

F. M. van der Veen

Institute of Psychology, Erasmus University Rotterdam,

PO Box 1738, 3000DR, Rotterdam, the Netherlands,

The Netherlands effects on mood and only minor effects on the reaction to the IAPS pictures were observed.

Conclusions A single dose of paroxetine inhibits emotional crying significantly. It is not sure what the underlying mechanism is. However, since there was no effect on mood and only minor effects on the response to emotional pictures, we postulate that paroxetine mainly acts on the physiological processes involved in the crying response.

Keywords Crying $\cdot$ Paroxetine $\cdot$ Serotonin $\cdot$ Emotion $\cdot$ Mood

\section{Introduction}

Crying is a uniquely human emotional response. The utility of crying for newborns, which seems functionally equivalent to the so-called separation calls of offspring of birds and mammals, is well established, but little is known about the functions, determinants, and consequences of crying in adults (Vingerhoets 2011; Vingerhoets et al. 2009). With respect to individual differences, factors, such as age, sex, attachment style, and personality (e.g., neuroticism and empathy), play a role (Vingerhoets 2011), but little is known about the underlying neurobiological mechanisms (Newman 2007). Crying is considered as a behaviour that typically occurs when attachment relations are broken and humans feel helpless and in need of support of others (Vingerhoets 2011).

Pathological crying, a condition frequently observed among neurological patients, can be successfully treated with selective serotonin reuptake inhibitors (SSRIs), suggesting that serotonin may modulate crying (Muller et al. 1999). Furthermore, studies have shown that tryptophan, which is the precursor of 5-HT, is reduced in women in the postpartum period (Maes et al. 2001), which is characterised by emotional lability and a high-crying proneness 
(Bailara et al. 2006). Finally, preliminary data suggest that being in love is accompanied by low 5-HT levels (Marazziti et al. 1999) and a greater crying proneness (Vingerhoets and van Assen 2009). Together, this evidence suggests that central 5HT levels might increase the threshold for crying. Therefore, we directly examined whether acute increases in central 5-HT levels by means of the SSRI paroxetine increases the crying threshold. To test this hypothesis, we designed a study in which participants watched two emotional movies on two different days and had to self-report their crying behaviour. On one day, the participants received paroxetine, and on the other day, they received placebo. It was hypothesised that selfreported crying would be reduced on the paroxetine day. Because there are some inconsistent findings suggesting that that SSRIs might influence the appraisal of emotional stimuli (Bruhl et al. 2010; Takahashi et al. 2005), we additionally examined the effect of paroxetine on the appraisal of emotional pictures. In this way, we could relate changes in crying behaviour to the possible effects on the appraisal of emotional stimuli and subsequent emotions which might underlie changes in emotional expression. Finally, we measured mood to examine whether preexisting mood or paroxetine induced mood changes were related to changes in crying behaviour. No direct effects on mood were expected.

\section{Method}

Participants

Participants were 25 healthy, young females recruited by means of advertisements. Inclusion criteria were: healthy females, free of medication (except for oral contraceptives) in the age-range of $18-27$ years $($ mean $=21.4$; standard error of mean $=0.4)$. The main reason to focus on young women is that we wanted to prevent floor effects. It is known that women in the fertile age range cry most frequently and we therefore expected to have the highest chance to measure an effect if we included only young women. Exclusion criteria were current somatic, neurological, and psychiatric disturbances and insufficient knowledge of the Dutch language to complete the questionnaires and mood self-reports. Health was checked with a short interview combined with a custom-made questionnaire. Participants were paid $€ 100$ for their participation. The study was approved by the Medical Ethics Committee of the Erasmus Medical Centre, Rotterdam, where this study was performed, and participants provided written informed consent.

\section{Design}

We applied a double-blind randomised placebo-controlled single-dose crossover design. Fifteen participants received $20 \mathrm{mg}$ paroxetine on the first day, and the other ten participants were administered paroxetine on the second day. Determination of the dosage was based on the usual dosage given to depressed patients and to allow comparability with other studies studying the effects of 5-HT on cognition and emotion using paroxetine (de Bruijn et al. 2006). In addition, it is known that $20 \mathrm{mg}$ paroxetine yields the maximal effect on central 5-HT levels, because of a maximal occupancy of the 5-HT transporter (Ruhe et al. 2009).

\section{Procedure}

Participants arrived between 9.00 and $10.00 \mathrm{~h}$ in the laboratory and were tested in a group of either 12 or 13 participants. The group of 12 participants watched 'Brian's Song' on the first day and 'Once Were Warriors' a week later, whereas the group of 13 participants watched the movies in the opposite order. Paroxetine and placebo, in the form of tablets, were administered orally. After administration of paroxetine or placebo, several questionnaires (see below) were completed. After a 5-h waiting period, which was implemented to achieve a maximal effect on central 5-HT levels (Kaye et al. 1989), participants first completed a mood questionnaire and subsequently watched the movie. After the movie, participants completed the crying questionnaire and judged emotional pictures (see below).

\section{Movies}

We used two emotional movies, i.e., 'Brian's Song' and 'Once Were Warriors', to induce crying behaviour. Both movies were successfully used in previous crying research by our research group. No specific instructions were given for watching the movie. We assessed crying behaviour by means of self-report. The outcome measure is the level of crying varying from 0 (no emotion) to 6 (teary eyes, sobbing, loud crying, body motions, and yelling), during a number of predetermined dramatic scenes.

\section{Questionnaires}

Mood was assessed at the beginning of the session and immediately before exposure to the movie on both days, using a visual analogue version of the profiles of mood scale (McNair et al. 1992).

Emotional pictures

Emotional pictures were derived from the International Affective Picture System (IAPS; Lang et al. 2005). Forty pictures were selected based on their standard norm scores. Ten pictures with a low-pleasure and low-arousal scores, ten pictures with a low-pleasure and high-arousal score, ten pictures with a high-pleasure and low-arousal score, and ten pictures with a high-pleasure and a high-arousal score were selected. Twenty of these pictures were used for the first 
session, and 20 were used for the second session; the order was balanced between subjects. Both sets of pictures thus consisted of five pictures of each of the four different stimulus categories. Pictures were projected on a screen for $6 \mathrm{~s}$ each. Each picture was immediately rated in terms of arousal and valence by means of the standardised selfassessment manikin (Lang 1980). After the rating was completed, the next picture was presented.

\section{Statistical analysis}

Ratings and mood were analysed using SPSS 16 for Windows. Subjective rating of crying behaviour was tested with a general linear model (GLM) for repeated-measures analysis with treatment (two levels; paroxetine vs. placebo) as withinsubjects factor and treatment order (two levels; paroxetine or placebo first) and movie order (two levels; 'Brian's Song' first or 'Once Were Warriors' first) as between-subjects factors. Average arousal and valence ratings were tested separately using a simple model with treatment (two levels; paroxetine vs. placebo) as within-subjects factor. We additionally evaluated the ratings for the subcategories of pictures in detail with arousal category (two levels; high arousal vs. low arousal), valence category (two levels; high pleasure vs. low pleasure) and treatment as within-subjects factors. Average subjective mood ratings as measured with the POMS were tested separately for the five subscales (anger, fatigue, depression, vigor, and tension) in a model with time (two levels; before or after movie) and treatment as within-subjects factor.

Table 1 lists the mean and standard error of mean scores of subjective ratings of the four categories of emotional pictures in subjects given paroxetine and placebo.

\section{Results}

\section{Crying behaviour}

Analysis of variance showed that paroxetine strongly affected the intensity of self-reported crying behaviour (main effect treatment, $F_{1,21}=16.9, P<0.0005, \eta^{2}=0.45$; see Fig. 1). For 'Brian's Song', mean $z$-score was $-0.41 \pm 0.29$ (mean \pm standard error of the mean) on the paroxetine day vs. $0.38 \pm 0.24$ on the placebo day. For 'Once Were Warriors', mean $z$-scores were $-0.47 \pm 0.16$ on the paroxetine day vs. $0.51 \pm 0.33$ on the placebo day. In addition, an interaction between treatment and movie order was found: $F_{1,21}=6.1, P<0.05, \eta^{2}=0.23$. Follow-up analyses showed that for the participants who saw 'Brian's Song' in the first session, the effect of treatment was stronger $(P<0.001$, onesided) and only marginally significant $(P=0.051$, onesided) for the opposite order (effect was in the same direction). The effect of treatment order did not interact with any of these effects.

\section{Emotional pictures}

In a first analysis, the average rating of all pictures in terms of arousal and valence was tested. No significant effects of paroxetine were found. In a second, more detailed, analysis, arousal and pleasure ratings of specific categories of the emotional pictures were evaluated with treatment, valence category and arousal category as within-subjects factors. In this analysis, we found, for arousal ratings, main effects of arousal category, $F_{1,24}=50.6, P<0.0005, \eta^{2}=0.68$, and valence category, $F_{1,24}=31.9, P<0.0005, \eta^{2}=0.57$, and two-way interactions between treatment and arousal category, $F_{1,24}=4.3, P<0.05, \eta^{2}=0.15$ and arousal and valence category, $F_{1,24}=9.8, P<0.0005, \eta^{2}=0.41$. As expected, pictures in the high-arousal category were rated as more arousing, and this effect was stronger for low-pleasure pictures. Treatment interacted with arousal ratings in different categories and follow-up paired $t$-tests showed that paroxetine only significantly decreased arousal ratings in the high-arousal/low-valence category.

For the pleasure ratings, we found main effects of arousal category, $F_{1,24}=30.3, P<0.0005, \eta^{2}=0.56$, and valence category, $F_{1,24}=151.7, P<0.0005, \eta^{2}=0.86$, and two-way interactions between treatment and valence category, $F_{1,24}=6.0, P<0.05, \eta^{2}=0.20$ and arousal and valence

Table 1 Mean and standard error of mean scores of the subjective ratings (arousal/pleasure) of the four categories of emotional pictures (high/low arousal, high/low pleasure) on the paroxetine and the placebo day

\begin{tabular}{|c|c|c|c|c|c|}
\hline \multicolumn{2}{|c|}{ Self-assessment manikin (SAM) } & \multicolumn{2}{|l|}{ High pleasure } & \multicolumn{2}{|l|}{ Low pleasure } \\
\hline & & High arousal & Low arousal & High arousal & Low arousal \\
\hline \multirow[t]{2}{*}{ Arousal } & Paroxetine & $2.7(.34)$ & $1.7(.17)$ & $3.6(.42)^{*}$ & $2.1(.21)$ \\
\hline & Placebo & $2.8(.13)$ & $1.7(.13)$ & $4.4(.34)$ & $2.2(.23)$ \\
\hline \multirow[t]{2}{*}{ Pleasure } & Paroxetine & $6.4(.18)^{*}$ & $6.1(.17)$ & $3.1(.23)$ & $4.7(.14)$ \\
\hline & Placebo & $6.8(.15)$ & $6.0(.17)$ & $2.7(.19)$ & $4.4(.16)$ \\
\hline
\end{tabular}

* Significant difference between paroxetine and placebo day $(P<0.05)$ 


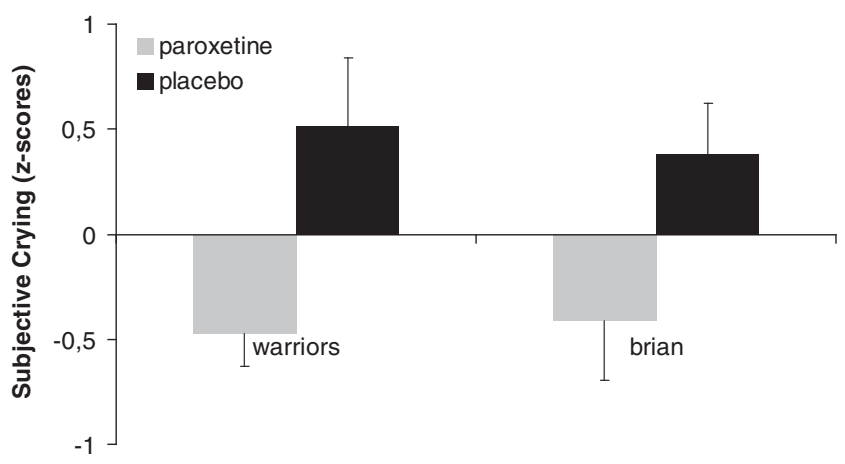

Fig. 1 Effect of paroxetine or placebo on subjective crying as expressed in $z$-scores ( \pm standard error of the mean) for 'Once Were Warriors' (warriors) and 'Brian's Song' (Brian)

category, $F_{1,24}=80.1, P<0.0005, \eta^{2}=0.77$. As anticipated, pleasure ratings were higher for the high-pleasure pictures, and this effect was stronger for pictures in the high-arousal category. Treatment interacted with pleasure ratings in different categories and follow-up paired $t$-tests showed that paroxetine only significantly decreased pleasure ratings in the high-arousal/high-valence category.

Given the treatment effect on arousal and pleasure ratings, one may wonder whether the altered appraisal of some emotional stimuli could be responsible for the reduced crying response to the movies, as observed in the paroxetine condition. To examine this, correlations between the picture ratings and crying were computed. This revealed a significantly positive correlation between the arousal score for the high-arousal/low-pleasure category in the paroxetine condition and crying $(r=0.52, P<0.01)$. In contrast, the valence scores in the high-pleasure/higharousal category did not show an association with crying. To summarise, paroxetine did not influence overall ratings of emotional pictures, but only in specific categories of pictures arousal and pleasure ratings were slightly lowered by paroxetine. The association between arousal scores and crying suggests a possible partly shared underlying process.

Mood

Mood was not affected by treatment or time. For none of the five subscales, significant main effects of treatment, time, or interactions between these two factors were found (all $P$ values $>0.15$ ).

\section{Discussion}

The main finding of the present study is that paroxetine reduces crying which clearly supports our hypothesis that 5HT is involved in crying. However, we are not yet certain about the underlying mechanism, since we found evidence that this effect, at least partially, can be attributed to the changed appraisal of emotional stimuli. Although only the arousal ratings of one subset of pictures and the pleasure ratings of another subset of pictures was affected, the effects were in the expected direction and especially the category of pictures, in which the arousal scores were affected, was most comparable to the movie scenes in terms of emotional content. In addition, arousal ratings were associated with crying behaviour only on the paroxetine day. This association suggests that a common process underlying both the crying in response to the movies and the appraisal of the pictures might be affected. One explanation is that the effect of paroxetine on crying was caused by reduced recognition of emotional film stimuli inducing the crying behaviour. However, this seems to be contradicted by earlier findings demonstrating that acute administration of a different SSRI (citalopram) enhanced recognition of fearful and happy facial expressions while leaving recognition of other emotional expressions intact (Harmer et al. 2003). A second possibility is that emotional impact of both the emotional film scenes and the emotional pictures is lowered by paroxetine. Recent neuroimaging findings support this notion. Citalopram decreased the amygdala response to aversive stimuli (Anderson et al. 2007) and fearful facial expressions (Murphy et al. 2009). This support, however, is indirect; moreover, the amygdala has been associated with both emotional expression (e.g., affective startle modulation; Angrilli et al. 1996) and evaluation of emotional stimuli. Finally, it should also be noted that the effects of paroxetine on arousal and pleasure ratings of the IAPS pictures in this study were small and the correlation with crying behaviour was modest, which suggests that the effect of paroxetine on crying behaviour is most likely not caused by a changed appraisal of emotional stimuli, but rather by its influence on other processes.

At first sight, the present effect of paroxetine on the evaluation of emotional pictures does not seem compatible with the results of previous studies (Bruhl et al. 2010; Takahashi et al. 2005), which failed to show an effect of different SSRIs on valence ratings of emotional pictures. It should be noted, however, that these studies failed to differentiate between subcategories of pictures in terms of arousal and that, in the present study, these effects only became manifest after differentiating. The effect of reduced arousal scores for high-arousal pictures and lower-valence scores for high-valence pictures suggests a blunting of affectivity, an established side effect of SSRIs (Opbroek et al. 2002; Price et al. 2009). A recent study (McCabe et al. 2010) further showed that the neuronal response to both aversive and rewarding stimuli was reduced after 1-week treatment with citalopram. However, the effects in the present study were acute and selective for specific categories of emotional pictures and therefore hard to compare with the more long-term and general effects of the McCabe et al. study. 
The effect of paroxetine on crying as an emotional expression seemed to contradict the results of other studies examining other emotional expressions in mice (Gomes et al. 2009) and humans (Grillon et al. 2007). More precisely, Grillon et al. showed that a single dose of citalopram facilitated the expression of fear in terms of an enhanced potentiating of a startle response, and Gomes et al. showed that acute effects of another SSRI (fluoxetine) increased anxiety-related behaviour. The differences between the specific SSRI used in the present and previous studies and the different doses used can possibly explain the somewhat inconsistent results in the various studies. Another possible explanation for these different effects could be the strong differences between the emotions involved in crying and the expression of fear. Fear is more directly involved in behaviour related to survival (fight/flight) and, in this way, is directly linked to action. The emotions involved in crying are not directly linked to action and are more related to passive, inactive states. Therefore, serotonin might not have an overall effect on emotional expressions, but might have differential effects on expressions with different functions. Crying is an emotional expression which can occur as the result of experiencing several different emotions. Tears of joy and sadness are clearly different, and, in this study, we examined crying behaviour as the result of two quite different movies. In one movie, crying behaviour is mostly caused by feelings of empathic sadness (Brian's Song) due to the sad and hopeless situation of the main character. In the second movie, emotions are more raw and violent and crying behaviour is mostly caused by feelings of an angry, horrified, or shocked type of sadness.

Since the relation between 5-HT and crying behaviour is not studied very extensively thus far, and precise relations with underlying emotions and different types of crying are unknown, the present study can be seen as a first starting point and the interesting results ask for further exploration concerning the effects of psychopharmacological agents on different types of crying and the underlying mechanisms.

Although the results are relatively clear and strong, the study has some limitations. First, the number of tested participants is relatively small, and we only tested female volunteers without controlling the hormonal cycle and use of oral contraceptives. Second, the number of used emotional pictures to measure changes in emotional appraisal was relatively small, and the present study only used subjective measures to quantify crying behaviour. Finally, although paroxetine is an SSRI, it might have affected other neurotransmitter systems directly or indirectly, and the reported effects therefore might have been at least partially caused by nonserotonergic mechanisms. Future studies should use more objective measures to quantify crying behaviour, aim to include a larger sample of volunteers, control hormonal status, and use more selective serotonergic manipulations.

In conclusion, this is the first study that shows a direct relation between central 5-HT levels and crying behaviour in healthy volunteers. It demonstrates that SSRIs have a major inhibitory effect on crying, which can have serious consequences for both the measured and experienced treatment effects of SSRIs. Further research is necessary to show whether the role of 5-HT is restricted to crying behaviour or whether it extends to other emotional expressions as well (e.g., smiling, laughing; see Mergl et al. 2003).In addition, the effects of SSRIs on the appraisal of emotional stimuli needs further clarification, with adequate attention for qualitatively different emotions. Future research should also focus on the possible effects of SSRIs on so-called sentimental or 'positive' crying, typically induced by movies depicting themes like self-sacrifice or altruism. Taken together, this would lead to a better insight into the underlying neurobiological mechanisms of this mysterious and important, but scientifically ignored and poorly understood human behaviour.

Acknowledgements This study was funded by the Tilburg University. This study was approved by the Medical Ethics Committee of the Erasmus Medical Centre, Rotterdam, where this study was performed, and participants provided written informed consent.

\section{Conflicts of Interest None}

Open Access This article is distributed under the terms of the Creative Commons Attribution Noncommercial License which permits any noncommercial use, distribution, and reproduction in any medium, provided the original author(s) and source are credited.

\section{References}

Anderson IM, Del-Ben CM, McKie S, Richardson P, Williams SR, Elliott R, Deakin JF (2007) Citalopram modulation of neuronal responses to aversive face emotions: a functional MRI study. Neuroreport 18:1351-1355

Angrilli A, Mauri A, Palomba D, Flor H, Birbaumer N, Sartori G, di Paola F (1996) Startle reflex and emotion modulation impairment after a right amygdala lesion. Brain 119(Pt 6):1991-2000

Bailara KM, Henry C, Lestage J, Launay JM, Parrot F, Swendsen J, Sutter AL, Roux D, Dallay D, Demotes-Mainard J (2006) Decreased brain tryptophan availability as a partial determinant of post-partum blues. Psychoneuroendocrinology 31:407-413

Bruhl AB, Kaffenberger T, Herwig U (2010) Serotonergic and noradrenergic modulation of emotion processing by single dose antidepressants. Neuropsychopharmacology 35:521-533

de Bruijn ER, Sabbe BG, Hulstijn W, Ruigt GS, Verkes RJ (2006) Effects of antipsychotic and antidepressant drugs on action monitoring in healthy volunteers. Brain Res 1105:122-129

Gomes KS, de Carvalho-Netto EF, Monte KC, Acco B, Nogueira PJ, Nunes-de-Souza RL (2009) Contrasting effects of acute and 
chronic treatment with imipramine and fluoxetine on inhibitory avoidance and escape responses in mice exposed to the elevated T-maze. Brain Res Bull 78:323-327

Grillon C, Levenson J, Pine DS (2007) A single dose of the selective serotonin reuptake inhibitor citalopram exacerbates anxiety in humans: a fear-potentiated startle study. Neuropsychopharmacology 32:225-231

Harmer CJ, Bhagwagar Z, Perrett DI, Vollm BA, Cowen PJ, Goodwin GM (2003) Acute SSRI administration affects the processing of social cues in healthy volunteers. Neuropsychopharmacology 28:148-152

Kaye CM, Haddock RE, Langley PF, Mellows G, Tasker TC, Zussman BD, Greb WH (1989) A review of the metabolism and pharmacokinetics of paroxetine in man. Acta Psychiatr Scand Suppl 350:60-75

Lang PJ (1980) Behavioral treatment and bio-behavioral assessment: computer applications. In: Sidowski JB, Johnson JH, Williams TA (eds) Technology in mental health care delivery systems. Ablex, Norwood, NJ, pp 119-137

Lang PJ, Bradley MM, Cuthbert BN (2005) International Affective Picture System (IAPS): Digitized photographs, instruction manual, and affective ratings (Tech. Rep. No. A-6). Gainesville: University of Florida, Center for Research in Psychophysiology

Maes M, Ombelet W, Verkerk R, Bosmans E, Scharpe S (2001) Effects of pregnancy and delivery on the availability of plasma tryptophan to the brain: relationships to delivery-induced immune activation and early post-partum anxiety and depression. Psychol Med 31:847-858

Marazziti D, Akiskal HS, Rossi A, Cassano GB (1999) Alteration of the platelet serotonin transporter in romantic love. Psychol Med 29:741-745

McCabe C, Mishor Z, Cowen PJ, Harmer CJ (2010) Diminished neural processing of aversive and rewarding stimuli during selective serotonin reuptake inhibitor treatment. Biol Psychiatry 67:439-445

McNair DM, Lorr M, Droppleman LF (1992) EdITS manual for the profile of mood states. Educational and Industrial Testing Service
Mergl R, Vogel M, Mavrogiorgou P, Gobel C, Zaudig M, Hegerl U, Juckel G (2003) Kinematical analysis of emotionally induced facial expressions in patients with obsessive-compulsive disorder. Psychol Med 33:1453-1462

Muller U, Murai T, Bauer-Wittmund T, von Cramon DY (1999) Paroxetine versus citalopram treatment of pathological crying after brain injury. Brain Inj 13:805-811

Murphy SE, Norbury R, O'Sullivan U, Cowen PJ, Harmer CJ (2009) Effect of a single dose of citalopram on amygdala response to emotional faces. Br J Psychiatry 194:535-540

Newman JD (2007) Neural circuits underlying crying and cry responding in mammals. Behav Brain Res 182:155-165

Opbroek A, Delgado PL, Laukes C, McGahuey C, Katsanis J, Moreno FA, Manber R (2002) Emotional blunting associated with SSRIinduced sexual dysfunction. Do SSRIs inhibit emotional responses? Int J Neuropsychopharmacol 5:147-151

Price J, Cole V, Goodwin GM (2009) Emotional side-effects of selective serotonin reuptake inhibitors: qualitative study. Br J Psychiatry 195:211-217

Ruhe HG, Booij J, v Weert HC, Reitsma JB, Franssen EJ, Michel MC, Schene AH (2009) Evidence why paroxetine dose escalation is not effective in major depressive disorder: a randomized controlled trial with assessment of serotonin transporter occupancy. Neuropsychopharmacology 34:999-1010

Takahashi H, Yahata N, Koeda M, Takano A, Asai K, Suhara T, Okubo Y (2005) Effects of dopaminergic and serotonergic manipulation on emotional processing: a pharmacological fMRI study. Neuroimage 27:991-1001

Vingerhoets AJ (2011) Tranen. Waarom mensen huilen. (Tears. Why humans cry). Amsterdam: Bert Bakker

Vingerhoets AJ, van Assen MA (2009) Love and tears. Poster presented at the Annual Meeting of the International Society for Research on Emotion, Leuven, Belgium

Vingerhoets AJ, Bylsma L, Rottenberg J (2009) Crying: a biopsychosocial phenomenon. In: Fögen $\mathrm{T}$ (ed) Tears in the GraecoRoman world. de Guyter, Berlin, pp 439-475 